\title{
Towards Feature-driven Goal Fulfillment Analysis A Feasibility Study
}

\author{
Avjot Garcha Singh ${ }^{1}$, Aida Omerovic ${ }^{2}$, Franck Chauvel $^{2}$ and Nicolas Ferry ${ }^{2}$ \\ ${ }^{1}$ University of Oslo, Oslo, Norway \\ ${ }^{2}$ SINTEF, Trondheim, Norway \\ avjotgs@ifi.uio.no, \{aida.omerovic, franck.chauvel, nicolas.ferry\}@sintef.no
}

Keywords: $\quad$ Model-based Analysis, Functional Analysis, Functional Fulfillment, Functional Overlap, Decision Support.

\begin{abstract}
Design of a system architecture normally refers to a pre-defined goal. We consider the goal as the desired (functional or non-functional) aspect that is achieved by providing functionalities or mechanisms that support it. For example, a security goal is supported by a set of security mechanisms. However, there are often several ways towards (partially or completely) achieving a goal, which we refer to as design alternatives. Each design alternative is composed of a subset of the mechanisms needed. Some mechanisms are more important than others, and it is not obvious to what degree the different design alternatives fulfill the goal. It can moreover often be difficult to see what combinations of the design alternatives would result in maximum coverage of the goal. We propose an approach to modeling and analysis of the goals of a system. The approach supports specification of the goal, specification of the design alternatives, a quantification of the degree of fulfillment, as well as a quantification of the degree of overlap with respect to the goal fulfillment across design alternatives. We also propose a visual representation of the degrees of fulfillment and the degrees of overlap. We have evaluated the approach on a case study, and the initial results indicate its feasibility. This paper presents the approach and the evaluation results. It also summarizes experiences and suggestions for further improvements.
\end{abstract}

\section{INTRODUCTION}

Everyone encounters decision making problems on a daily basis. The common denominator for all decision making problems is that the decisions are often made based on satisfaction for where the decision criterion is maximized or minimized.

In a perfect decision making scenario a decision alternative stands out by satisfying all decision criteria. However, it is quite rare that a decision alternative satisfies all decision criteria. In most of the cases, the decision maker selects a decision alternative based on preferences and priorities of the decision criteria. In that manner, it is often necessary to perform a thorough analysis of a complex decision making scenario. "Decision analysis can thus be defined as the process and methodology of identifying, modeling, assessing, and determining an appropriate course of action for a given decision problem. This process often involves a wide array of tools and the basic approach is generally to break the problem down into manageable and understandable parts that the decision maker can comprehend and handle. It is then necessary to take these smaller elements and reconstitute them into proper solution for the larger original problem" (Ravindran, 2007).

When planning design of a system, security goals need to be taken into account as early as possible. For some design decisions, the alternatives and their impacts need to be considered long before the implementation. The current state of the art provides several security threat and risk oriented approaches such as for example cause-consequence analysis (Nielsen, 1971), attack trees (Schneier, 1999), CRAMM (Barber and Davey, 1992), OCTAVE (Alberts and Davey, 2004), and CORAS (Lund et al., 2011). These approaches focus to a high degree on protecting assets or goals of systems being analyzed, from their known threats and risks. The threats and the risks are however not necessarily known at the early stages. There is lack of approaches which facilitate security analysis by explicitly modeling which features and measures can generally contribute to the security goal of a system being planned, without a priori knowledge of the risks.

Imagine an enterprise wanting to make changes to their information system architecture in order to improve the security. The enterprise has for instance al- 
located five possible decision alternatives that might be beneficial regarding security of their information system. How do they know which security features and mechanisms (or measures) would be appropriate for achieving the goal? Are these mechanisms practically feasible in combination? Provided the decision alternatives are described at a high level, can we refine them and thus identify which of the mechanisms (that ideally are desired as specified by the goal) they cover? If the mechanisms are of different importance, can we quantify to what degree they fulfill the goal? If several decision alternatives can be combined, can we provide decision support choosing the preferred combination, such that the goal is fulfilled to highest possible degree?

In this paper, we tackle these needs by proposing an approach to functional fulfillment analysis. By functional fulfillment analysis we mean the analysis of 1) degree of fulfillment of a predefined (functional and non-functional) goal, and 2) degree of overlap between the decision alternatives with respect to the goal. The degree of fulfillment reflects the coverage of features and mechanisms related to the various decision alternatives with respect to the goal. By functionalities or features we mean the partial goals or properties of the target of analysis. By mechanisms we mean the implementable measures that support the achievement of the partial goals. By overlap we mean the association, interplay, and relationship between the various decision alternatives. The approach to modeling offers notation, terminology, and guidance for expressing the degree of fulfillment and the degree of overlap. The focus on the functional aspects of the approach is due to the need of making the functionalities and features first class citizens, and decomposing the goal via the functionalities to the mechanisms for achieving them. A functional analysis thus allows a broader perspective on a goal, as compared to a pure quality (non-functional) analysis.

The approach has been evaluated in a case study targeting a system called SensApp (Mosser et al., 2012). The input to the case study were comprehensive architecture design models of SensApp, as well as a high-level specification of five design alternatives aiming at improvement of SensApp security. In addition to two analysts, two domain experts with thorough knowledge of SensApp, were present throughout the case study, and were actively involved in the development of the models for functional fulfillment analysis. The evaluation indicates that the functional fulfillment analysis provides useful information regarding the performance of the decision alternatives. We were able to model functional capabilities of the goal, and the decision alternatives, as well as to fully

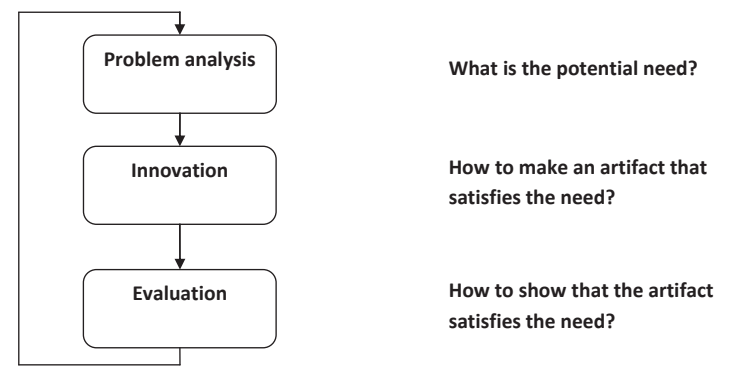

Figure 1: Method for technology research (adopted from Solheim and Stølen (Solheim and Stølen, 2007)).

analyze both the degree of fulfillment and the degree of overlap. The comprehensibility and the expressiveness of the models seemed to be satisfactory in the context of the case study, while correctness and certainty of the models need further enhancements of the approach. More evaluation is furthermore needed in order to address the threats to validity and reliability, but we believe that the approach could be useful in the context of decision making where multiple decision alternatives can be selected and combined. Based on our initial experiences, we suggest further work on uncertainty handling, process guidance, tool support development, and more empirical evaluation.

The paper is structured as follows: Section 2 summarizes our objective, success criteria, and the research method. In Section 3 we briefly present the background state of the art, and explain main constructs of feature modeling. Section 4 briefly presents our approach in general terms. Section 5 presents how the approach was applied on a case study. In Section 6 , we elaborate on the degree to which the success criteria have been achieved, and discuss the threats to validity and reliability. In Section 7, main conclusions and directions for future work are summarized.

\section{RESEARCH METHOD}

Our objective has been to provide a practically useful decision support for model-based and goal-oriented design. To this end, we model goals in such a manner that the mechanisms needed for achieving them are explicit. A part of the objective is moreover to be able to analyze the degree to which the functionalities and the mechanisms contribute to fulfillment of a goal. Additionally, we needed support for comparing the design alternatives and expressing their mutual similarity or overlap in terms of the functionalities and mechanisms supporting the goal under analysis. Considering the need of the users to correctly develop and use the models, our initial success criteria for the 
approach have included:

- correctness of the models,

- expressiveness of the models, and

- comprehensibility of the approach to the users.

The correctness implies that the set of models are unbiased as well as sufficiently certain for the purpose intended, and should substantiate and support the decisions made by the decision maker. In that manner, uncertainties must be taken into account. The uncertainties involved must be reported and managed properly. The expressiveness implies that the models can include all the aspects that are relevant for the selection of the decision alternatives. The comprehensibility implies that the models are easy to understand by the users. The models should moreover facilitate a common understanding considering the decision making problem in question. The approach assumes that the modelers have the relevant domain knowledge on the system under analysis. Normally, an analyst and several domain experts would collaborate on development of the models. Indicators for comprehensibility by the domain experts or the decision makers include involvement of the stakeholders, agreement upon the models, ability to use the models correctly, correct interpretation, and consistent estimates.

Our research was based on an iterative process consisting of problem analysis, innovation, and evaluation, as illustrated by Figure 1 (Solheim and Stølen, 2007). During the problem analysis phase, SensApp, i.e. the target of the analysis was modeled in terms of its architecture, risks, costs of the various decision alternatives, and the system quality. The need for comparing the functional coverage and overlap between the five different decision alternatives, was identified and the above presented success criteria were characterized.

The innovation phase consisted of designing a solution based on the identified needs. In our case, the approach to modeling functional and technical aspects, analyzing them with respect to coverage of the goal, and visualizing the goal fulfillment, was proposed.

Our evaluation was conducted by collaborating with two domain experts in SensApp, and performing the modeling and analysis of the security-relevant functionalities of SensApp. Based on the initial target system description and the UML (Alhir, 1998) system models of SensApp (which were input to our analysis), the analysts were to a certain degree able to propose an initial model decomposing the goal. During the rest of the evaluation, the approach was further developed in close collaboration with the domain experts during two workshops with duration of four hours in total. The domain experts were also involved in reviewing the needs and requirements related to the approach. Thus, the approach has, to a certain degree, been developed and evaluated in parallel. Finally, the approach to modeling in the decision making method was evaluated with respect to a pre-defined set of success criteria.

\section{BACKGROUND}

This section places our work in the context of the state of the art. We summarize the modeling approaches we have relied on, before presenting feature modeling in more detail.

\subsection{The Context}

This work has been motivated by the need to distinguish between decision alternatives based on their goal achievement through functionalities and measures. Often, decision support considers aspects such as quality, risks, and cost. The functional properties are however not analyzed as a first class citizen. Establishing a mindset which focuses on functionalities supporting a goal, provides a perspective which is oriented towards external properties of a system and the mechanisms for achieving them. Importantly, we do not omit the non-functional aspects, but include them in the modeling when needed. Our approach relies on Dependency Views (DVs) (Omerovic, 2012) and the feature models (Thüm et al., 2014) in modeling the functional coverage and similarity of the decision alternatives, with respect to a pre-defined goal. The adopted modeling techniques are well-known and chosen with objective to be familiar and comprehensible to non-technical users.

We make use of feature modeling to decompose the goal into subgoals, features and mechanisms. We moreover use the propagation models from DVs within the PREDIQT method (Omerovic, 2012) to model degree of fulfillment. Feature diagrams have a hierarchical tree structure containing features, characteristics, and aspects associated with the object in question (Thüm et al., 2014). Feature diagrams are useful in the sense that they provide insight into the various features and functionalities implemented by the decision alternatives. Similarly to the DVs, this approach quantifies the degree of fulfillment. It however distinguishes from the DVs by focusing mainly on the functional properties of a goal.

With respect to the feature models, our models are enriched by the annotated weights and a propagation 

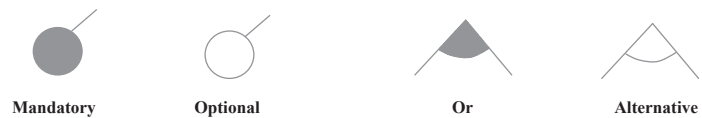

Figure 2: Syntax of feature diagrams.

model. With respect to the DVs, our models are not concerned with degree of fulfillment of each subgoal or mechanism (assuming that it is fully fulfilled), but only its importance which is normalized and propagated as in a DV. The operators (AND, OR and XOR, mandatory, optional) from the feature models are applied and the propagation model extended to handle them as well. We moreover quantify the degree of overlap between the decision alternatives, and propose how both the degree of fulfillment and the degree of overlap can be visualized.

\subsection{Feature Modeling}

A feature diagram represents the features, characteristics, and aspects associated with the target of analysis (Thüm et al., 2014). Feature diagrams have a hierarchical tree structure containing either mandatory or optional features. The various sub-features are often distinguished with or-, and-, or alternativerelationships. Feature diagrams are useful in the sense that they provide insight into the features and functionalities implemented by the various decision alternatives. A design alternative is depicted by a selected set of features which are decomposed with respect to a goal.

Figure 2 illustrates the syntax of feature diagrams. Mandatory and optional features are explicitly distinguished and represent an and-relationship. All mandatory sub-features must be selected in an andrelationship. The or-relationship describes features that are required by the parent-feature. In that manner, the sub-features of an or-relationship are indifferent and at least one sub-feature must be selected (Thüm et al., 2014). The alternative-relationship describes alternative features that are not required by the parentfeature, and exactly one sub-feature has to be selected (Thüm et al., 2014). A feature that is not described in a feature diagram and specified by an alternativerelationship can still be selected as long it fulfills the purpose given by the parent-feature. In summary, the relationships include:

- Alternative: Not required features and exactly one feature must be selected

- And: Mandatory and optional features

- Or: Required features and at least one feature must be selected

\section{OUR SOLUTION}

The functional fulfillment analysis expresses the degree of fulfillment of a goal, and the degree of overlap between the decision alternatives with respect to the coverage of the goal. While the degree of fulfillment quantifies the coverage of the features and mechanisms supporting achievement of the goal, the overlap expresses the similarity of two possible decision alternatives with respect to the features and mechanisms supporting the goal. In this section, we briefly present the approach in general; first the overall process, second the modeling and finally the analysis part of it.

\subsection{Process of the Approach}

The six stage process of the approach is depicted by Figure 3. The input to the process are the design models of the target of the analysis, and a description of the goal. In Phase 1, the ideal solution (objective) is modeled resulting in a feature model. In Phase 2, a feature model is deduced for each decision alternative (DA) from the model representing the objective. Next, the arcs on the objective model are annotated with weights expressing their importance. The decision alternative specific feature diagrams thus inherit the weights from the relevant parts of the objective diagram. Next, the degrees of fulfillment and the degrees of overlap are calculated for all decision alternatives. Finally, we visualize the results in a unified view and preform selection by searching for the set of decision alternatives having maximum fulfillment and minimal overlap. Phases 1, 2 and 3 need to be done in collaboration between an analyst and domain experts. Phases 4 and 5 are based on algorithms which can be automated. The final phase is use of the models, and left to a decision maker.

\subsection{Modeling}

The approach to modeling offers notation, terminology, and guidance for expressing the degree of fulfillment and the degree of overlap. Our approach makes use of feature diagrams to represent the objective and the various decision alternatives. A feature diagram representing the objective reflects the ideal functionality of the target system under analysis. In that manner, the feature diagrams representing the various decision alternatives will be sub-graphs of the feature diagram representing the overall objective. This is the case because only the relevant elements (i.e, those already expressed through the objective model) need to 


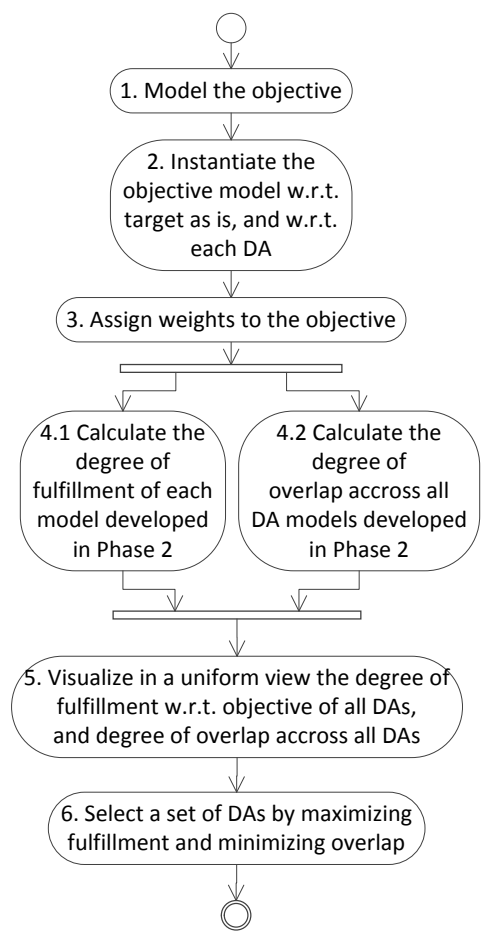

Figure 3: The process to be undergone.

be included in the instantiation with respect to the decision alternatives.

The feature diagrams are expected to be developed by the analyst in close collaboration with the domain experts. First of all, a feature diagram representing the ideal functionality of the target system should be developed. The feature diagram should contain subgoals, features and finally mechanisms (in the form of leaf nodes) that fulfill the overall objective. Furthermore, feature diagrams representing the various decision alternatives should be developed. The feature diagrams are then annotated with weights which enable the calculation of the degree of fulfillment and the degree of overlap achieved by the decision alternatives. In the following, we explain in detail the calculation behind the degree of fulfillment and the degree of overlap.

\subsection{The Degree of Fulfillment}

In this section, we present a formalization of the calculation behind the degree of fulfillment. The calculation of the degree of fulfillment of a feature diagram is considered by comparing the nodes with an initial feature diagram representing the overall objective. A feature diagram is a set of nodes $N$, where each node $n$ is assigned a weight $w$. A node denotes a relevant feature related to the decision alternative in question. If $n$ is a part of an and-relationship, then the assigned weight $w$ of $n$ should be taken into account by dividing $w$ by the sum of weights assigned to all sibling nodes in the initial feature diagram. A node is a sibling to another node if they both share the same immediate parent node. In that manner, all sibling nodes are part of the same sub-graph.

Furthermore, we need to take into account the constraints provided by the alternative- and orrelationship. If the node in question is a part of an alternative- or or-relationship in the initial feature diagram, then we do not divide $w$ by the sum of weights assigned to all sibling nodes. In that case, we only consider the node in question and divide the weight $w$ by itself. Finally, we do not distinguish between mandatory and optional nodes, since the difference between mandatory and optional features is already ensured and maintained through the assigned weights.

As an example, we let the initial feature diagram FD (corresponding to feature diagram representing the overall objective) contain the following nodes;

$$
F D=n_{i}, \ldots, n_{j} \text { where } i, j \in N
$$

Similarly, we let the feature diagram in question FD' (corresponding to feature diagram representing a decision alternative) contain the following nodes;

$$
F D=n_{k}, \ldots, n_{z}
$$

In the following we summarize the general rules for calculating the degree of fulfillment:

- If $n_{i}$ is a part of an and-relationship, then the calculated degree of fulfillment of the node in question will be $\frac{w_{i}}{w_{\text {sum }}}$, where $w_{\text {sum }}$ denotes the total weight of all sibling nodes.

- If $n_{i}$ is a part of an alternative- or or-relationship, then the calculated degree of fulfillment of the node in question will be $\frac{w_{i}}{w_{i}}$. In that manner, the degree of fulfillment achieved by a specific node in an alternative- or or-relationship will correspond to one.

For aggregating the total degree of fulfillment for the whole feature diagram, we provide the following pseudo code for tree traversal:

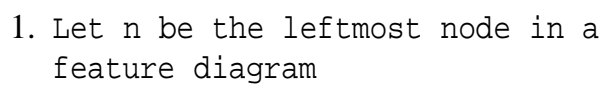

2. If $\mathrm{n}$ is a part of an and-relationship: $\frac{w}{w_{\text {sum }}}$ where $\mathrm{w}$ denotes the weight of $\mathrm{n}$ and $w_{\text {sum }}$ denotes the total weight of all sibling nodes

3. If $\mathrm{n}$ is a part of an alternative- or or-relationship: $\frac{w}{w}$ where $\mathrm{w}$ denotes the weight of $\mathrm{n}$ 
4. Apply the above steps for every child node of $n$

5. The degree of fulfillment of $\mathrm{n}$ will be multiplied with the degree of fulfillment achieved by each child

6. The degree of fulfillment achieved by the leaf-nodes taking part in an and-relationship will be summed

\subsection{The Degree of Overlap}

The degree of fulfillment tells nothing about the overlap between the decision alternatives. The intuition of the degree of overlap is to identify decision alternatives with similar features and functionality. By considering the degree of overlap, we can exclude a decision alternative that might provide the same features and mechanisms as another decision alternative. The degree of overlap quantifies the extent to which two decision alternatives provide the same features and supporting mechanisms. The degree of overlap follows the same calculation as the degree of fulfillment presented in the previous section. While the degree of fulfillment is calculated with the objective as reference model, the degree of overlap is obtained by having the various decision alternatives as reference models.

\section{EVALUATION BASED ON A CASE STUDY}

This section first presents a brief description of the target of analysis. Secondly, we provide an account of the instantiation of the approach on the case. The input to the case were architecture models of the target of analysis (Singh, 2014), as well as a characterization of the five decision alternatives.

\subsection{Case Description}

The case study was conducted on a small system called SensApp (Mosser et al., 2012). SensApp is an open-source service-based application used to store and exploit data collected by the Internet of Things (IoT). SensApp can register sensors, store the data collected by the sensors and notify registered clients with relevant data and information (Mosser et al., 2012). The main stakeholders involved in the context of SensApp are: sensor architect, service provider, data miner, and third party application. The sensor architect is responsible for registering sensors. The sensors push data to the application, which indirectly trigger the sending of a notification when relevant data is pushed. The service provider is responsible for the operations and maintenance of the sensors. The data miner can query stored data, while a third party application in addition has the ability to register for notifications when relevant data is pushed.

The case study was conducted in an information security context with the objective of improving the security of SensApp. Two analysts and two domain experts participated in the entire case study. The following above mentioned decision alternatives (DAs) were analyzed:

- DA A: Change in infrastructure

- DA B: Change of topology

- DA C: Change of licenses

- DA D: Change of location

- DA E: Update software

All decision alternatives were specified in more detail and considered as realistic alternatives for security improvement. Change in infrastructure means change in the technical architecture needed for the functioning of the service provided by SensApp. Change of topology means change in the configuration of the technical base needed for the functioning of the service provided by SensApp. Change of licenses involves upgrading or purchasing enterprise and commercial software licenses for information security purposes. By change of location we mean geographical relocation of the infrastructure, the platform, and the environment that SensApp is based upon. Updating the current software version of SensApp involves implementation of various security mechanisms in the already existing solution of SensApp.

\subsection{Modeling Security Subgoals, Features, and Mechanisms}

All developed diagrams focus on security relevant features. We used FeatureIDE (Thüm et al., 2014) to develop our feature diagrams. FeatureIDE is an Eclipse-based tool for development of feature diagrams. Figure 4 illustrates the security relevant features associated with the ideal functionality of SensApp. The domain experts identified in particular four security domains that could be improved in terms of the objective of enhancing the security of SensApp: (1) education, (2) policies, (3) risk management, and (4) infrastructure. Furthermore, Figure 4 describes security mechanisms as leaf-nodes that could be implemented in order to fulfill the overall objective. We 


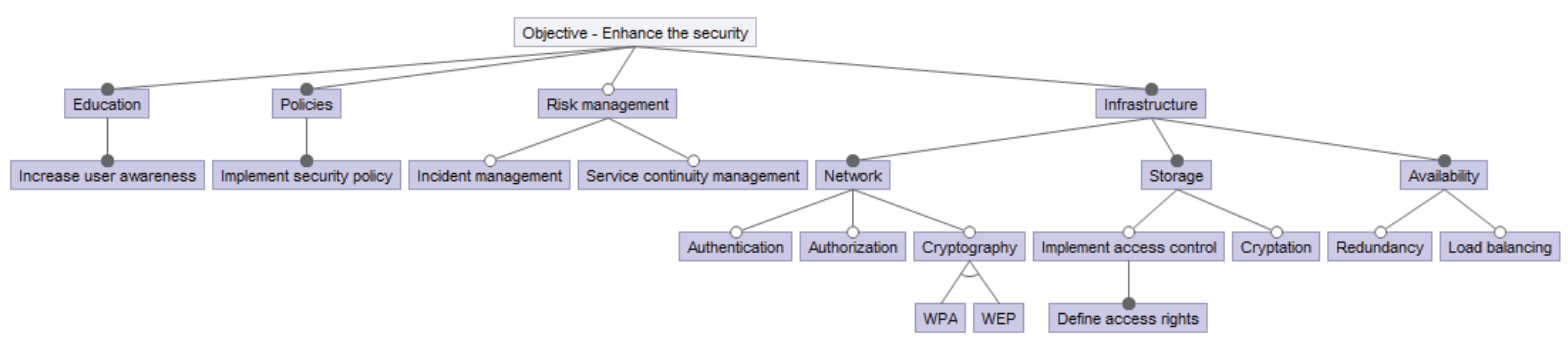

Figure 4: Objective - Enhance the security.

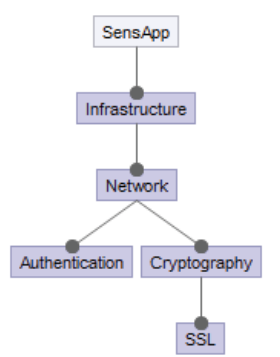

Figure 5: Current security of SensApp.

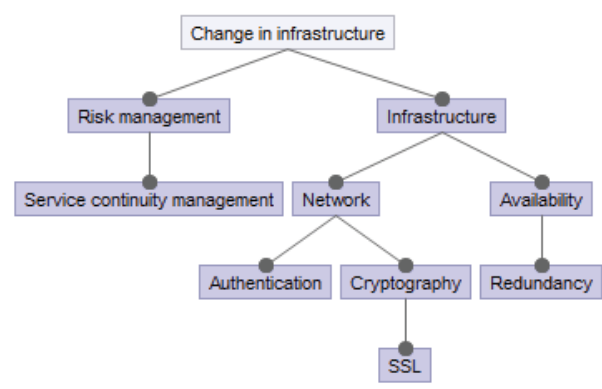

Figure 6: Security relevant features associated with decision alternative A.

will use Figure 4 as a reference model during evaluation of our method for calculating the degree of fulfillment.

Figure 5 expresses the current security of SensApp. As shown, the existing version of SensApp has implemented authentication and cryptography mechanisms.

Figure 6 shows the feature diagram expressing the result of implementation of decision alternative A change in infrastructure. Thus, Figure 6 illustrates the various security relevant features and mechanisms associated with SensApp after the implementation of decision alternative $\mathrm{A}$. The implementation of decision alternative A will bring improvement in availability and redundancy. Change in infrastructure will in addition introduce authentication and cryptography mechanisms that will improve the security within the network. Moreover, decision alternative A will implement security-related mechanisms that will introduce service continuity management.

Figure 7 shows the feature diagram expressing the

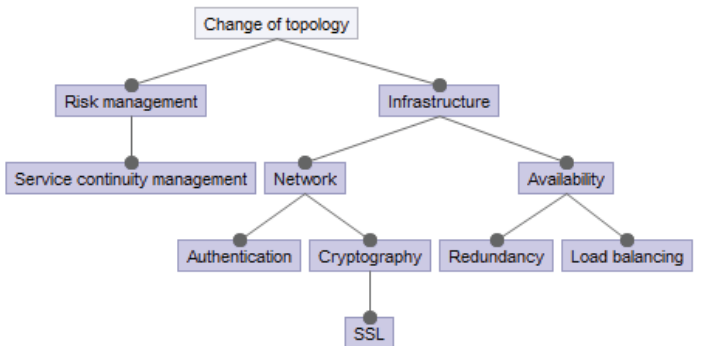

Figure 7: Security relevant features associated with decision alternative $B$.

result of implementation of decision alternative B change of topology. In that manner, Figure 7 illustrates the various security relevant features associated with SensApp after the implementation of decision alternative B. Similar to decision alternative A, the decision alternative $\mathrm{B}$ will bring improvement in availability. In addition to redundancy, decision alternative B will provide load balancing. Decision alternative B will also provide the implementation of both authentication and cryptography mechanisms.

Figure 8 shows the feature diagram expressing the result of implementation of decision alternative $\mathrm{C}$ change of licenses. Figure 8 expresses the various security relevant features associated with SensApp after the implementation of decision alternative C. By upgrading or purchasing enterprise and commercial software licenses, there will be a need for increasing user awareness and implementing security policies. Moreover, decision alternative $\mathrm{C}$ will implement various security mechanisms for securing the network and the storage. In addition, it is possible to purchase licenses from external information security vendors for monitoring and management of security-related incidents.

Figure 9 shows the feature diagram expressing the result of implementation of decision alternative D change of location. Relocation of Information Technology services often introduce legal issues, therefore there will be a need of increasing user awareness and implementing security policies. Furthermore, decision alternative $\mathrm{D}$ will bring improvement in network security by implementing various authentication and 


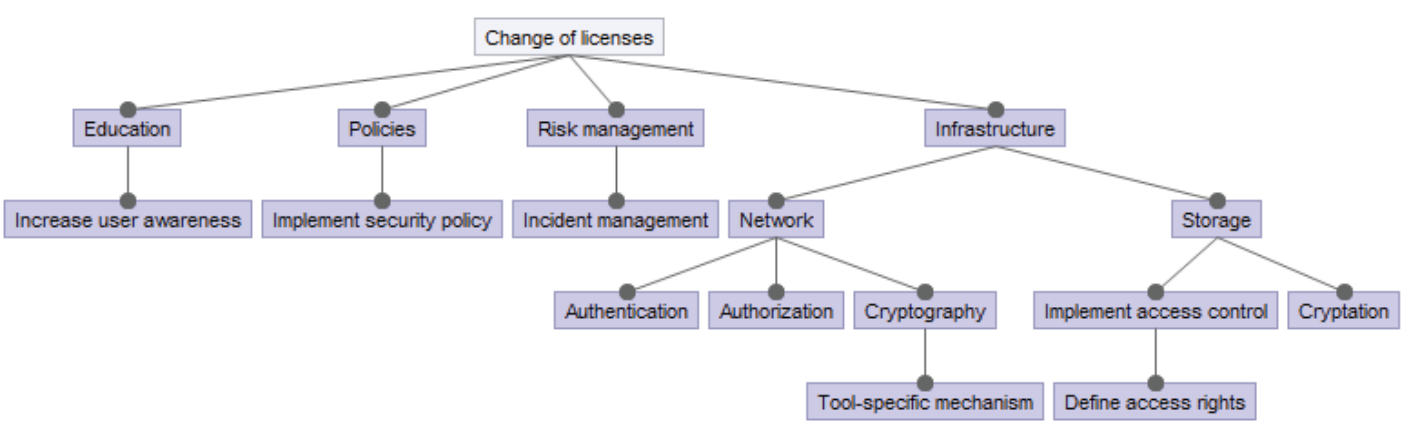

Figure 8: Security relevant features associated with decision alternative C.

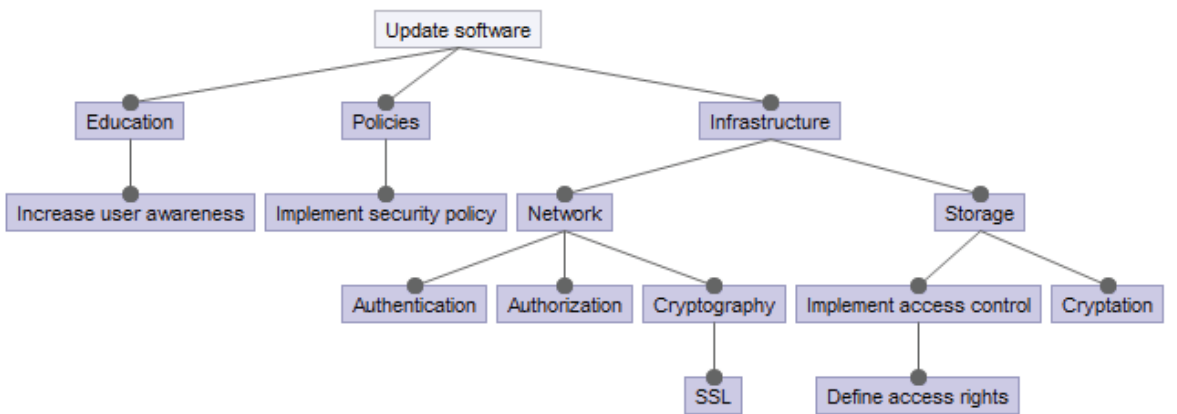

Figure 10: Security relevant features associated with decision alternative E.

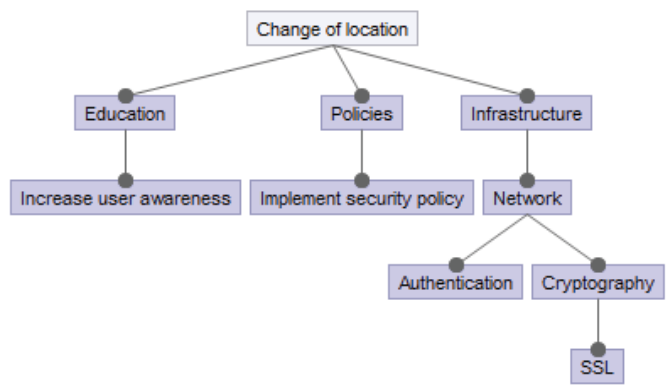

Figure 9: Security relevant features associated with decision alternative D.

cryptography mechanisms.

Figure 10 shows the feature diagram expressing the result of implementation of decision alternative $\mathrm{E}$ - update software. The decision alternative $\mathrm{E}$ will implement various security mechanisms for providing more robust security of the network and the storage.

\subsection{Modeling the Degree of Fulfillment}

The degree of fulfillment reflects the coverage of security features related to the various decision alternatives. We assigned weights to all arcs on the feature diagram representing the ideal functionality (the objective) of SensApp (Figure 12). A weight denotes the importance of a security feature with respect to the parent node. In this case, the security features were assigned weights according to the scale specified in

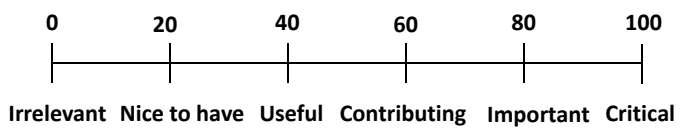

Figure 11: Scale for assigning weights.

Figure 11. The scale was agreed upon and defined by the domain experts. Assigning weights was done based on a top-down approach, i.e., starting from the root node, its children nodes are weighted with respect to the root. The weights were then assigned to the children nodes at the next level until reaching the leaves and having all arcs of the model (representing the objective) annotated.

The feature diagrams representing the various decision alternatives inherit the weights assigned to the objective (Figure 12). This is possible since the feature diagrams representing the various decision alternatives are sub-graphs of the feature diagram representing the objective of SensApp. Feature diagrams containing the objective of SensApp and decision alternative A with assigned weights are presented in Figure 12 and 13, respectively.

The sub-features of infrastructure in Figure 12 represent three mandatory sub-groups namely network, storage, and availability. It is important to point out that the weight assigned to infrastructure represents the importance of infrastructure given the overall objective, while the weights assigned to the various sub-features of infrastructure represent the impor- 


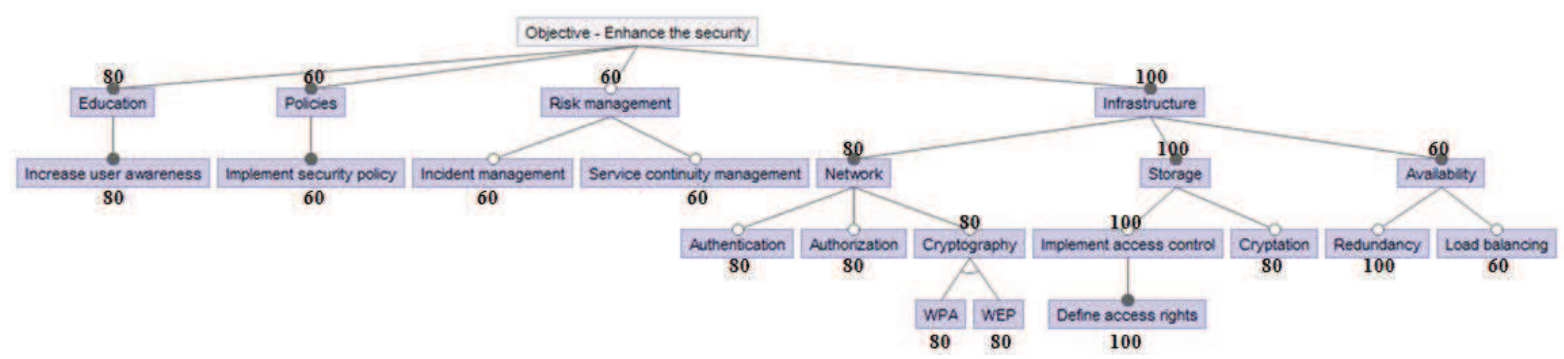

Figure 12: Objective with assigned weights.

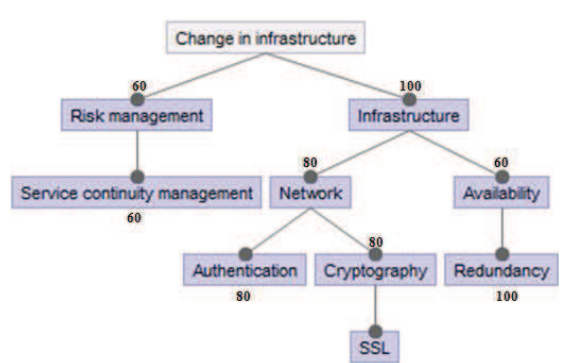

Figure 13: Decision alternative A with assigned weights.

tance given the parent-feature. Network is therefore important while storage is critical given the infrastructure of the information system in question. The sub-features of an alternative-relationship should be assigned the same weight, since they all are equally representative for the parent-feature. In that manner, both WEP and WPA are important security relevant features given the feature of cryptography. The difference between mandatory and optional features is ensured and maintained through the assigned weights.

For each decision alternative, the weights on the corresponding diagram propagate to the resulting overall security of SensApp. We perform aggregation of the degree of fulfillment by normalizing all contributions in such a manner that the objective achieves the degree of fulfillment equal to one, while the decision alternatives score in terms of a fraction relative to the objective. Thus, the degree of fulfillment achieved by decision alternative A was obtained as shown in Equation 3.

The calculation is based on a top-down approach, where the weight of each node is aggregated by the principle of normalization. In that manner, decision alternative A has achieved 0.226 degree of fulfillment in the following manner. The first term in the calculation represents the node of education, where the denominator represents the sum of the weights in the first level in Figure 12. The numerator is zero since the node of education is not represented by decision alternative A. Similar reason yields for the second term in the calculation. The third term represents the node of risk management, where the numerator represents the assigned weight and the denominator represents the sum of the weights in the first level of the feature diagram in Figure 12. Further, the node of risk management involves implementation of incident management and service continuity management. Decision alternative A does not provide incident management, and we therefore only take into account service continuity management in the calculation. The numerator in the aggregated term represents the assigned weight of service continuity management, while the denominator represents the sum of the assigned weight of both incident management and service continuity management.

The calculation is similar for the node representing infrastructure. However, it is important to notice that only one of the sub-features in an alternativerelationship has been taken into account in the calculation. In that manner, the calculation is only based on one of the assigned weights to the alternativerelationship consisting of WPA and WEP. Since the sub-features of an alternative-relationship should be assigned the same weight, the denominator should represent the assigned weight of the parent-feature.

This approach was similarly undergone to calculate the degree of fulfillment achieved by decision alternatives B-E. The corresponding calculation for the overall decision alternatives (DAs) results in the following fulfillment values (where value of 1 implies a perfect fulfillment, a value of 0 implies no fulfillment, while the values between o and 1 imply percentages of the fulfillment of the objective):

- DA B: 0,257

- DA C: 0,917

- DA D: 0,541

- DA E: 0,717

\subsection{The Degree of Overlap}

The degree of fulfillment tells nothing about the overlap between the decision alternatives. We use the approach for calculating the degree of fulfillment to 


$$
\frac{0}{300}+\frac{0}{300}+\frac{60}{300}\left(\frac{60}{120}\right)+\frac{100}{300}\left(\frac{80}{240}\left(\frac{80}{240}+\frac{0}{240}+\frac{80}{240}\left(\frac{80}{80}\right)\right)+\frac{0}{240}+\frac{60}{240}\left(\frac{100}{160}+\frac{0}{160}\right)\right) \approx 0.226
$$

Propagation of the degree of fulfillment regarding decision alternative A

Table 1: Degree of overlap between the decision alternatives.

\begin{tabular}{|c|c|c|c|c|c|}
\hline & DA A & DA B & DA C & DA D & DA E \\
\hline DA A & & 0.9 & 0.099 & 0.417 & 0.123 \\
\hline DA B & 1 & & 0.099 & 0.417 & 0.123 \\
\hline DA C & 0.357 & 0.357 & & 1 & 1 \\
\hline DA D & 0.357 & 0.357 & 0.565 & & 0.707 \\
\hline DA E & 0.357 & 0.357 & 0.80 & 1 & \\
\hline
\end{tabular}

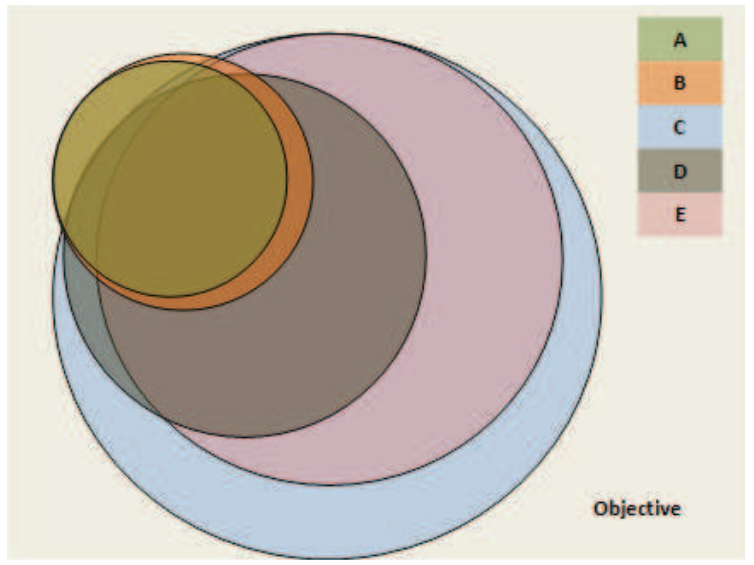

Figure 14: Visual representation of the degree of fulfillment and the degree of overlap.

obtain the degree of overlap between the various decision alternatives. Namely, we calculate the degree of overlap between decision alternatives $\mathrm{X}$ and $\mathrm{Y}$ by having $\mathrm{X}$ (rather than the objective) as reference model for calculation of degree of fulfillment of Y. The degree of overlap expresses to which extent two decision alternatives $\mathrm{X}$ and $\mathrm{Y}$ provide the same security-related mechanisms. Table 1 presents the resulting degree of overlap between our decision alternatives $\mathrm{A}$ through $\mathrm{E}$. The top row in Table 1 indicates the reference models.

The intuition of the degree of overlap is to identify decision alternatives with similar subgoals, features, and mechanisms. According to Table 1, the securityrelated mechanisms provided by decision alternative A are also provided by decision alternative B. However, decision alternative B provides load balancing as well (see Figure 7). In that manner, it would be reasonable to exclude decision alternative $\mathrm{A}$.

Figure 14 visualizes the degree of fulfillment and the degree of overlap obtained by the decision alternatives from the SensApp case. The size of the elements in Figure 14 depicts the degree of fulfillment, while the placement of the elements depicts to what extent the various decision alternatives overlap with respect to security features and functionality. According to Figure 14, the security-related mechanisms provided by decision alternatives $\mathrm{D}$ and $\mathrm{E}$ are covered by decision alternative $\mathrm{C}$. This can also be observed from Table 1.

\section{DISCUSSION}

According to Runeson and Höst (Runeson and Höst, 2009): "The validity of a study denotes the trustworthiness of the results, to what extent the results are true and not biased by the researchers' subjective point of view". There was a large number of contextual factors influencing our research, such as: the case study setting, the research method, the target system under analysis, and the participants involved. Our research was highly dependent on subjective judgments provided by the domain experts. As major threat we consider the uncertainty of the models. The domain experts provided estimates based on their experience. In that manner, the validity of the models relies on the confidence to the expert judgments. There exists state of the art that we could adopt for uncertainty handling. Uncertainty handling has, for example, been reviewed in (Omerovic et al., 2012). In DVs, uncertainty is handled based on intervals (Omerovic and Stølen, 2011).

The in-depth technological background of the domain experts has to some degree limited the threats to validity. We achieved some triangulation by using multiple sources of evidence in order to strengthen our results. The sources of evidence during the model development have included: target system descriptions, UML system models, and presentation slides. During the workshops, the domain experts were actively involved in characterization of the weight scale and the model development. This indicates a common understanding of the target and the approach.

It is important to emphasize that our case study was based on a small information system with limited complexity. In that manner, it may be argued that SensApp was not a representative case for our research. However, the size and the complexity of SensApp were suitable for evaluation of the feasibility of the approach. Moreover, our evaluation was conducted with respect to security only, which in turn 
is a restriction considering our definition of the functional fulfillment analysis. A broader trade-off analysis considering several goals simultaneously, would have been even more realistic. Another important fact is that the case study mainly aimed at testing feasibility of the approach. The models developed have not been verified; their main role was 1) to provide an example which demonstrates application of the approach and 2) to facilitate further improvement and evaluation of the approach. Therefore, the models should not be considered as correct, nor should the case study results be regarded as a security analysis of SensApp.

One missing part we experienced (which a risk analysis would have included) is the notion of the acceptance level regarding the degree of fulfillment. We could namely not tell whether our best decision alternative (or combination of thereof) was good enough, since it did not fully reach the objective. Another missing feature of the method was explicit optimization with respect to degree of fulfillment and overlap. Yet another assumption we have made when proposing the degree of overlap, is that several decision alternatives can be combined. Decision alternatives may however not always be compatible, in which case it will not make sense to consider degree of overlap.

The approach has been proposed and evaluated in the context of security. Rather than applying the existing security threat and risk oriented approaches (e.g., attack trees (Schneier, 1999), CRAMM (Barber and Davey, 1992), OCTAVE (Alberts and Davey, 2004), and CORAS (Lund et al., 2011)) we aimed at explicitly modeling how security features and measures contribute to the overall security goal. As such, our approach is more oriented towards early design of a system, rather than protection of an existing one. A challenge of the early design is however lack of empirical data for modeling, particularly estimation of the weights.

Our success criteria are concerned about correctness, expressiveness, and comprehensibility. As argued above, correctness needs further evaluation and explicit uncertainty handling. We were able to model the objective and all decision alternatives, express all subgoals, functions and mechanisms in the models, as well as to analyze both degree of fulfillment and the degree of overlap. The scale proposed seemed to provide sufficient intuition to assign estimates to the objective. Hence, there are indications of expressibility of the models. Moreover, the active participation of the domain experts and the fact that they were able to agree upon the final models, indicates comprehensibility of the approach. Main challenge in developing of the approach was the balancing of the success criteria. Practical usefulness requires that the models are sufficiently informative and correct, at the same time as being easy to understand for a non-expert user. Therefore, we have for the sake of simplicity put some restrictions on the granularity of the models, and the amount of the information being visualized in the last step of the approach. Although our results indicate practical feasibility of the approach, further evaluation is needed in order to assess validity and reliability of the approach.

\section{CONCLUSIONS AND FUTURE WORK}

We have put forward an approach to functional fulfillment analysis. By functional fulfillment analysis we mean the analysis of 1) degree of fulfillment of a goal, and 2) degree of overlap between the decision alternatives with respect to the goal. The degree of fulfillment expresses the coverage of the measures supporting the goal, while overlap expresses the similarity between the various decision alternatives with respect to the measures involved. By considering the degree of fulfillment and the degree of overlap in a uniform view, we can select a combination of decision alternatives which includes most influential security measures, at the same time as overlaps are avoided. Overlapping measures are particularly relevant to avoid when repetition imposes additional costs.

The approach has been evaluated in a case study targeting a system called SensApp. The evaluation indicates feasibility in the sense that the approach could be applied on a case study and provide useful information regarding the performance of the decision alternatives. We were able to model functional capabilities of the goal, and the decision alternatives, as well as to fully analyze both the degree of fulfillment and the degree of overlap. Additionally, we were able to visualize the overall performance of the decision alternatives by employing our approach to visualizing the decision alternatives.

The comprehensibility and the exspressivness of the models seemed to be satisfactory in the context of the case study, while correctness of the models need further enhancements of the approach. The largest concern is the lack of confidence in the estimates. The main threat to our findings is that they are dependent and based on subjective matters. More evaluation is furthermore needed in order to address the threats to validity and reliability, but we believe that the approach could be useful in the context of decision making where multiple decision alternatives can be selected and combined. 
Based on our initial experiences, we propose further work on extending the method with acceptance levels and compatibility of decision alternatives, uncertainty handling, process guidance, tool support, and more empirical evaluation (involving systems with larger complexity and from other domains). Future work should also address the robustness of the calculations involved in our approach. By robustness we mean the extent to which the degree of fulfillment of the decision alternatives would change if the input estimates change. Related to this, we also intend to address sensitivity of the approach in general and impact of the decision alternatives in particular.

\section{ACKNOWLEDGMENTS}

This work has been partially supported by the MODAClouds project (Grant Agreement FP7318484) and the NESSoS network of excellence both funded by European Commission within the 7th Framework Programme. It has also been partially supported by the DIAMONDS project funded by the Research Council of Norway.

\section{REFERENCES}

Alberts, C. J. and Davey, J. (2004). OCTAVE criteria version 2.0. Technical report CMU/SEI-2001-TR-016, Carnegie Mellon University.

Alhir, S. S. (1998). UML in a Nutshell - A Desktop Quick Reference. OReilly \& Associates, Inc., Sebastopol, first edition edition.

Barber, B. and Davey, J. (1992). The use of the ccta risk analysis and management methodology cramm in health information systems. In In 7th International Congress on Medical Informatics, MEDINFO92.

Lund, M. S., Solhaug, B., and Stølen, K. (2011). Model-Driven Risk Analysis - The CORAS Approach. Springer.

Mosser, S., Fleurey, F., Morin, B., Chauvel, F., Solberg, A., and Goutier, I. (2012). Sensapp as a reference platform to support cloud experiments: From the internet of things to the internet of services. In Management of resources and services in Cloud and Sky computing workshop, Timisoara. IEEE.

Nielsen, D. S. (1971). The cause/consequence diagram method as basis for quantitative accident analysis. Technical report RISO-M-1374, Danish Atomic Energy Commission.

Omerovic, A. (2012). PREDIQT: A Method for Modelbased Prediction of Impacts of Architectural Design Changes on System Quality. Doctoral Dissertation, Faculty of Mathematics and Natural Sciences, University of Oslo, Oslo.
Omerovic, A., Karahasanovic, A., and Stølen, K. (2012). Uncertainty handling in weighted dependency trees: A systematic literature review. In Dependability and Computer Engineering: Concepts for SoftwareIntensive Systems. IGI Global.

Omerovic, A. and Stølen, K. (2011). A practical spproach to uncertainty handling and estimate acquisition in model-based prediction of system quality. International Journal on Advances in Systems and Measurements, 4(1-2):55-70.

Ravindran, A. R. (2007). Operations Research and Management Science Handbook. CRC Press,, Boca Raton.

Runeson, P. and Höst, M. (2009). Guidelines for conducting and reporting case study research in software engineering. Department Computer Science, Lund University.

Schneier, B. (1999). Attack Trees: Modeling Security Threats. Dr. Dobbs Journal.

Singh, A. G. (2014). Towards a Decision Support Method for Trade-off Analysis Considering Cost, Risk, and Quality. MSc Dissertation, Faculty of Mathematics and Natural Sciences, University of Oslo, Oslo.

Solheim, I. and Stølen, K. (2007). Technology Research Explained. SINTEF. Technical Report A313, Oslo.

Thüm, T., Kästner, C., Benduhn, F., Meinicke, J., Saake, G., and Leich, T. (2014). FeatureIDE: An Extensible Framework for Feature-Oriented Software Development. Science of Computer Programming. 